\title{
Uncooled, Millimeter-Scale Atomic Magnetometers with Femtotesla Sensitivity
}

\author{
John Kitching, Svenja Knappe, W. Clark Griffith, Jan \\ Preusser, Vladislav Gerginov, Peter D. D. Schwindt, \\ Time and Frequency Division \\ NIST \\ Boulder, CO USA
}

\author{
Vishal Shah, Ricardo Jimenez-Martinez \\ Department of Physics \\ University of Colorado \\ Boulder, CO USA
}

\begin{abstract}
We summarize recent results at NIST to develop highperformance yet highly miniaturized magnetic sensors based on atomic vapors confined in microfabricated alkali vapor cells. These sensors currently achieve sensitivities in the range of a few tens of femtotesla in a $1 \mathrm{~Hz}$ bandwidth in detection volumes of about $1 \mathrm{~mm}^{3}$. They require no cooling and only a small amount of heating, making them ideal for deployment in remote, power starved locations.
\end{abstract}

\section{INTRODUCTION}

The measurement of weak magnetic fields or field gradients enables the detection and evaluation of a variety of electromagnetic sources, from metallic objects to neural currents. While there exists a diverse spectrum of magnetic sensors based on a variety of physical effects, the "gold standard" for precision measurement of DC magnetic fields is the superconducting quantum interference device (SQUID) [1]. Commercial versions of these sensors typically have sensitivities in the range of $1 \mathrm{fT} / \sqrt{\mathrm{Hz}}$, with increased noise at frequencies below about $10 \mathrm{~Hz}$.

One major application for SQUID-based magnetic sensors is the measurement of biomagnetic fields [2]. The human brain, for example, produces magnetic signals with a magnitude just outside the head of about $1 \mathrm{pT}$. The human heart produces signals about 100 times larger when measured just outside the chest. These magnetic signals can be used for diagnosis and treatment of a variety of medical conditions including cardiac ischemia and epilepsy. In addition, there exists the tantalizing possibility that magnetically detected brain signals could be deciphered to monitor what a person is thinking. For example, recent research has shown that monkeys with electrodes implanted in their brains have been able to control a prosthetic limb with a precision required to feed themselves [3]. Magnetic sensors could measure very similar signals without the need for electrodes inside the body and may provide a much safer, lower-cost alternative to electrode-based encephalography.

Commercial biomagnetic instrumentation already exists, with magnetocardiograph (MCG) machines and magnetoencephalograph (MEG) machines being the main players. These systems have volumes typically of several cubic meters, because of the large cryostats required to cool the SQUIDs down to their operating temperature. They are also very expensive to purchase and operate because of the complexity of the overall system. We propose that if the sensitivity of chip-scale atomic magnetometers described here could be improved to the $\mathrm{fT} / \sqrt{\mathrm{Hz}}$ range, they could be used to great advantage as a replacement for SQUID-based sensors in these instruments. The ultimate impact would be much wider deployment of biomagnetic instrumentation at much lower cost.

In this paper, we review recent results from our group focused on the development of chip-scale atomic magnetometers, with a particular view toward biomagnetic instrumentation. The goals of this work are to develop lowcost, miniaturized sensors with sensitivities well below 1 $\mathrm{pT} / \sqrt{\mathrm{Hz}}$. In particular, we trace the progress from the earliest instruments demonstrated in 2004 with a sensitivity of 40 $\mathrm{pT} / \sqrt{\mathrm{Hz}}$ [4] to the most recent instruments incorporating flux concentrators with a sensitivity near $10 \mathrm{fT} / \sqrt{ } \mathrm{Hz}[5]$.

\section{Chip-Scale Atomic Magnetometers}

\section{A. Basic Physics}

Chip-scale atomic magnetometers are based on the effects of magnetic fields on the orientation of spin-polarized atoms in the vapor phase [6]. While either nuclear or electron polarization can be used, most commercial atomic magnetometers are based on the electron polarization in alkali (or alkali-like) atoms, which have a single valence electron. The valence electron has both angular momentum and a magnetic moment and hence precesses about a magnetic field at the Larmor frequency, which in the low-field limit is proportional to the magnetic field. By measuring the Larmor frequency, the magnetic field can therefore be obtained.

In a typical instrument, atoms of $\mathrm{Cs}, \mathrm{Rb}$ or $\mathrm{K}$ are confined in a sealed glass vapor cell. The atoms are optically pumped using a circularly-polarized light source resonant with an 
optical transition in the atoms. Atoms absorb light with the specific polarization and reradiate it isotropically, becoming polarized along the light beam direction in the process. The atoms are then driven with a magnetic field oscillating near their Larmor frequency; the atoms begin to precess about the DC magnetic field. Near resonance, the phase shift between the Larmor precession and the drive field is roughly proportional to the frequency difference between the two. The atomic precession is measured using the absorption of a probe light field; this absorption depends on the orientation of the atoms, and hence the power transmitted through the atomic ensemble is modulated at the precession frequency of the atoms.

Most commercial atomic magnetometers therefore have four main components: a light source (usually a lamp excited by an RF discharge), a vapor cell containing the atoms, a pair of coils to generate the drive field, and a photodetector to detect the transmitted probe. In most commercial magnetometers, the pump and the probe are the same light field.

\section{B. Microfabricated Design}

Chip-scale atomic magnetometers take advantage of micromachined components to achieve a high degree of miniaturization. The central component is the micromachined alkali vapor cell $[7,8]$. It is fabricated by first etching a hole, about $1 \mathrm{~mm}$ across, in a $1 \mathrm{~mm}$-thick silicon wafer using a standard MEMS etching process. Both deep reactive ion etching and wet chemical etching have been shown to work well. A piece of glass approximately $0.2 \mathrm{~mm}$ thick is anodically bonded to one side. This "preform" is then placed inside a vacuum chamber, and the alkali metal, along with an appropriate buffer gas, is deposited into the opening in the silicon. Finally a second glass wafer is anodically bonded to the top of the silicon wafer, sealing the materials inside the cell.

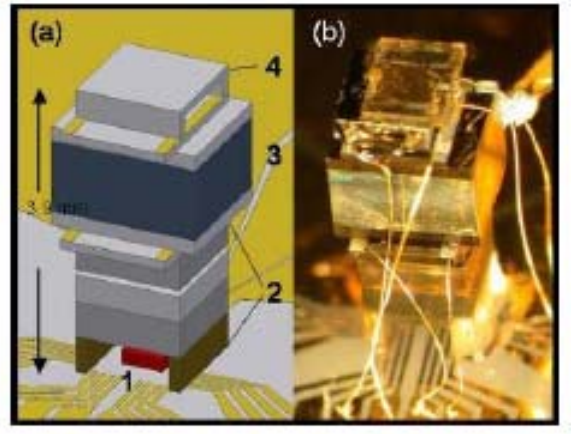

Fig. 1: The first chip-scale atomic magnetometer demonstrated at NIST in 2004. From ref. [4].

The vapor cells can be assembled into a stacked structure to form the magnetometer sensor head. The first such structure demonstrated at NIST in 2004 [4] is shown in Fig. 1. In this structure, a laser (1) is mounted onto a baseplate that allows electrical signals to be transmitted to and from the sensor. On top of the laser sits an optics assembly (2), which serves to collimate, attenuate and polarize the light emitted from the laser. The cell (3) is placed on top of the optics assembly between a pair of heaters. Finally a photodiode (4) is placed on top of the entire structure to collect the light transmitted through the atomic sample. More details of this structure can be found in Ref. [4].

The sensitivity of this instrument was approximately 40 $\mathrm{pT} / \sqrt{\mathrm{Hz}}$ in the frequency band from $1 \mathrm{~Hz}$ to $100 \mathrm{~Hz}$. The bandwidth was approximately $100 \mathrm{~Hz}$.

This early device was far from being optimal. First, it was based on a measurement of a magnetically-sensitive hyperfine transition in the atoms rather than a Larmor resonance. While this configuration has certain advantages (reduced heading error and no dead zones, for example), it requires the presence of a gigahertz oscillator and has limited prospects for improved sensitivity.

We therefore constructed a second sensor head based on a measurement of the Larmor frequency directly. This device is shown in Fig. 2 and described more fully in reference [9]. The main change over the device in Fig. 1 is the addition of a pair of coils around the cell. These coils allowed the modulation of the magnetic field in the vicinity of the vapor cell and hence the excitation of the Larmor precession.

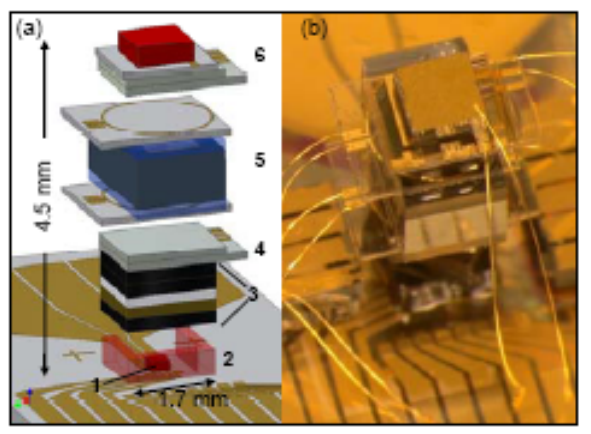

Fig. 2: Chip-scale atomic magnetometer based on interrogation of the Larmor precession. From ref. [9].

The sensitivity of this instrument was about $6 \mathrm{pT} / \sqrt{\mathrm{Hz}}$ in a frequency band between $10 \mathrm{~Hz}$ and $1 \mathrm{kHz}$.

\section{Chip-Scale Magnetometers With Suppressed Spin Exchange} BROADENING

The performance of atomic magnetometers is limited ultimately by collisions between alkali atoms. This can be understood in the following way. If one wants to obtain the best signal-to-noise ratios, it is better to use a large number of atoms. This means operating the magnetometer at a high alkali density (and hence high cell temperature). However, when the density of alkali atoms gets very high, spin-exchange 
collisions between the atoms cause the Larmor resonance to broaden and the sensitivity to degrade. In this limit, increasing the alkali density no longer improves the sensitivity. The best atomic magnetometers operating in this spin-exchange limited regime have sensitivities in the range of a few $\mathrm{fT} / \sqrt{\mathrm{Hz}}$ $[10,11,12]$.

Recently, it was pointed out that considerably improved sensitivity could be obtained in a regime in which spinexchange collisions were suppressed [13]. This regime, known as the "spin-exchange relaxation free" or SERF regime, occurs at very high alkali densities and very low magnetic fields. Atomic magnetometer sensitivities below $1 \mathrm{fT} / \sqrt{ } \mathrm{Hz}$ can be obtained using this technique $[14,15]$.

We therefore carried out measurements of the sensitivity of a magnetometer in the SERF regime based on a microfabricated vapor cell. The experimental configuration for these measurements is shown in Fig. 3 and described more fully in Ref. [16].

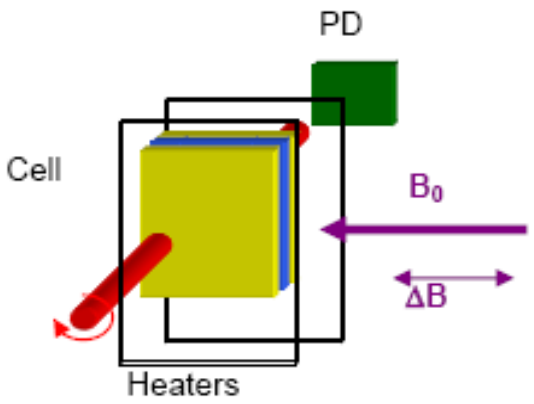

Fig. 3: Magnetometer in the SERF regime based on a microfabricated alkali vapor cell. From ref. [16].

The vapor cell is placed in a magnetically shielded environment and is interrogated with a circularly-polarized free-space laser beam. The cell is heated to a high temperature $\left(150^{\circ} \mathrm{C}\right)$ and a weak magnetic field is applied. Near zero field, the atoms become spin-polarized along the direction of the laser beam and the absorption is reduced. At a non-zero field, the atoms are reoriented with a component perpendicular to the laser beam and a reduced component along the laser beam. This causes an increased absorption.

In the SERF regime, a very narrow resonance of approximately $70 \mathrm{nT}$ was observed. This resonance could be measured with a signal to noise of $\sim 10^{6}$ in a $1 \mathrm{~Hz}$ bandwidth, resulting in a sensitivity of $70 \mathrm{fT} / \sqrt{\mathrm{Hz}}$. The bandwidth of this sensor was approximately $100 \mathrm{~Hz}$.

A final improvement in the sensitivity was obtained by the use of flux concentrators [5]. These components, made of high-permeability material, concentrate the magnetic flux into the vicinity of the vapor cell, increasing the field in this region at the expense of larger device volume. We note, however, that the power required to run the sensor could still be quite small, since only the small sensor head would need to be heated.

Flux concentrators based on ferrite rods are shown next to a micromachined alkali vapor cell in Fig. 4. These concentrators were designed to increase the field at the cell location by about a factor of 20 . They performed as expected but added a small amount of noise caused by fundamental thermal properties of the concentrator material itself. The sensitivity at a frequency of $100 \mathrm{~Hz}$ was improved by a factor of 10 as compared to the sensor performance without the concentrators.

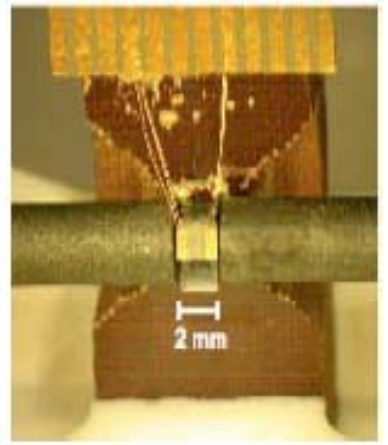

Fig. 4: Magnetometer in the SERF regime incorporating flux concentrators. From ref. [5].

\section{SENSITIVITY COMPARISON}

The sensitivity of each of the magnetometers described above in shown in Fig. 5. It can be seen that the SERF magnetometers $(\mathrm{C}$ and $\mathrm{D})$ perform considerably better than those limited by spin exchange collisions (A and B). The SERF magnetometers are in the range of what is required for biomagnetic measurements.

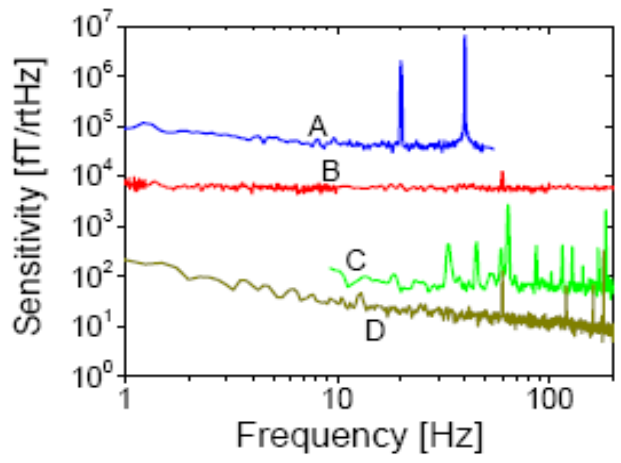

Fig. 5: Comparison of magnetometer sensitivities as a function of Fourier frequency. A: Chip-scale atomic magnetometer shown in Fig. 1; B: Chip-scale atomic magnetometer shown in Fig. 2; C: SERF magnetometer shown in Fig. 3; D: Magnetometer with flux concentrators shown in Fig. 4.

\section{CONCLUSION}

We have described the spectrum of atomic magnetometers developed at NIST over the last few years based on 
microfabrication techniques. These magnetometers feature high sensitivity, small size, low power operation and ease of manufacturing, potentially leading to low cost. They are enabled not only by the use of micromachining techniques but also by the use of low-power diode lasers, such as verticalcavity surface-emitting lasers.

The sensitivity of these instruments is beginning to put them within reach of a number of biomagnetic applications such as MCG and MEG. In addition, several other important applications appear viable including magnetic anomaly detection, perimeter monitoring, nuclear magnetic resonance detection and the characterization of magnetic fields in space.

\section{ACKNOWLEDGMENT}

We acknowledge funding from DARPA, SERDP and NIST. This work is a contribution of NIST, an agency of the US government, and is not subject to copyright.

\section{REFERENCES}

[1] R. L. Fagaly, "Superconducting quantum interference device instruments and applications", Rev. Sci. Instrum., vol. 77, page 101101 (2006).

[2] K. Sternickel and A. I. Braginski, "Biomagnetism using SQUIDs: status and perspectives," Supercond. Sci Technol., vol. 19, pages S160-S171, 2006.

[3] M. Velliste, S. Perel, M. C. Spalding, A. S. Whitford, and A. B. Schwartz, "Cortical control of a prosthetic arm for self-feeding," Nature vol. 453, pages 1098-1101, 2008.

[4] P. D. D. Schwindt, et al., "Chip-scale atomic magnetometer," Appl. Phys. Lett., vol. 85, pages 6409-6411, 2004.

[5] W. C. Griffith, R. Jimenez-Martinez, S. Knappe, J. Kitching, and V. Shah, "Miniature atomic magnetometer integrated with flux concentrators," Appl. Phys. Lett., vol. 94, page 023502, 2009.

[6] H. G. Dehmelt, "Modulation of a Light Beam by Precessing Absorbing Atoms," Phys. Rev., vol. 105, page 1924, 1957.

[7] L. A. Liew, S. Knappe, J. Moreland, H. Robinson, L. Hollberg, and J. Kitching, "Microfabricated alkali atom vapor cells," Appl. Phys. Lett., vol. 84, pages 2694-2696, 2004.

[8] S. Knappe, V. Gerginov, P. D. D. Schwindt, V. Shah, H. G. Robinson, L. Hollberg, and J. Kitching, "Atomic vapor cells for chip-scale atomic clocks with improved long-term frequency stability," Opt. Lett., vol. 30, pages 2351-2353, 2005.

[9] P. D. D. Schwindt, et al., "A chip-scale atomic magnetometer with improved sensitivity using the Mx technique," Appl. Phys. Lett., vol. 90, page 081102, 2007.

[10] D. Budker, D. F. Kimball, S. M. Rochester, V. V. Yashchuk and M. Zolotorev, "Sensitive magnetometry based on nonlinear magneto-optical rotation," Phys. Rev. A, vol. 62, page 043403, 2000.

[11] S. Groeger, G. Bison, J. L. Schenker, R. Wynands and A. Weis, "A high-sensitivity laser-pumped Mx magnetometer," Eur. Phys. J. D, vol. 38, pages 239-247, 2006.

[12] E. B. Alexandrov, M. V. Balabas, A. S. Pasgalev, A. K. Vershovskii and N. N. Yakobson, "Double resonance atomic magnetometers: From gas discharge to laser pumping," Laser. Phys., vol. 6, pages 244-251, 1996.

[13] W. Happer and H. Tang, "Spin-exchange shift and narrowing of magnetic resonance lines in optically pumped alkali vapors," Phys Rev. Lett., vol. 31, pages 273-276, 1973.

[14] J. C. Allred, R. N. Lyman, T. W. Kornack, and M. V. Romalis, "Highsensitivity atomic magnetometer unaffected by spin-exchange relaxation," Phys. Rev. Lett., vol. 89, page 130801, 2002.
[15] I. K. Kominis, T. W. Kornack, J. C. Allred, and M. V. Romalis, "A subfemtotesla multichannel atomic magnetometer," Nature, vol. 422, pages 596-599, 2003.

[16] V. Shah, S. Knappe, P. D. D. Schwindt, and J. Kitching, "Sub-picotesla atomic magnetometry with a microfabricated vapor cell," Nat. Phot., vol. 1, pages 649-652, 2007. 